\title{
Control of Metabolism by Central and Peripheral Clocks in Drosophila
}

\author{
Amita Sehgal
}

\begin{abstract}
Drosophila is a powerful system for the molecular analysis of circadian clocks, providing the first account of how such a clock is generated. It is also proving to be an excellent model to dissect the neural basis of circadian behavior. In addition, clocks are located in peripheral tissues in flies, but much less is known about these clocks and about the physiological processes they control. This chapter describes the use of Drosophila for understanding the circadian control of metabolism. While a clock in the fat body is critical for metabolic function, it is clear that neuronal clocks are also involved. Indeed, synchrony between these clocks is important for reproductive fitness. A complex interplay between circadian and metabolic signals is indicated by the finding that metabolic pathways can even impact rest:activity rhythms controlled by the brain clock. Drosophila may be an optimal system to dissect the nature of these interactions and their importance for organismal fitness and life span.
\end{abstract}

Genetic analysis of circadian rhythms started with the isolation of the period (per) mutants in the fruit fly, Drosophila melanogaster, followed by isolation of the per gene in the mid 1980s (Bargiello et al. 1984; Jackson et al. 1986; Konopka and Benzer 1971; Reddy et al. 1984; Zehring et al. 1984). Subsequent studies identified the per partner, timeless (tim), and the transcriptional feedback loop that we now know lies at the heart of the clock mechanism in all species (Sehgal et al. 1994, 1995). In the Drosophila loop, the Clock (CLK) and cycle (CYC) transcriptional activators promote expression of per and tim mRNA during the mid to late day but are repressed by feedback activity of PER-TIM in the late night and early morning. Regulated expression and activity of clock proteins in this loop are sustained through post-translational mechanisms, in particular the action of multiple kinases and phosphatases (Zheng and Sehgal 2008, 2012).

Contrary to expectations that clocks would be localized largely, if not exclusively, in the brain, analysis of Drosophila per showed that it was expressed in multiple tissues throughout the body (Liu et al. 1988; Saez and Young 1988). Indeed, use of a reporter in which per was fused to firefly luciferase showed that

\footnotetext{
A. Sehgal $(\bowtie)$

Perelman School of Medicine, University of Pennsylvania, Philadelphia, PA, USA

e-mail: amita@mail.med.upenn.edu

(C) The Author(s) 2016

P. Sassone-Corsi, Y. Christen (eds.), A Time for Metabolism and Hormones,

Research and Perspectives in Endocrine Interactions,

DOI 10.1007/978-3-319-27069-2_4
} 
per was expressed cyclically in most tissues. Analysis of isolated tissues revealed that luciferase activity continued to cycle in the absence of neural connections or systemic signals, indicating the presence of tissue-autonomous clocks (Plautz et al. 1997). Subsequent studies showed that the degree of autonomy varied from tissue to tissue. The Malphigian tubules or fly kidneys, for instance, appeared to be completely autonomous, such that they retained their own "timing" even when transplanted into a host that was synchronized to a different day:night cycle (in other words, a different time zone; Giebultowicz et al. 2000). On the other hand, the clock in the prothoracic gland, which drives a circadian rhythm of eclosion (hatching of adult flies from pupae) in Drosophila, is "slave" to the "master clock in the brain (Myers et al. 2003). Thus, the brain clock is required for eclosion rhythms as well as for maintenance of the prothoracic clock (Myers et al. 2003). In addition, central nervous system signals, in particular the neuropeptide Pigment Dispersing Factor (PDF), modulate the clock in pheromone-producing oenocytes, which regulate mating (Krupp et al. 2013).

The emerging pattern is that of a network of clocks that control many aspects of physiology and depend upon neural function to varying extents. The question is the extent to which Drosophila can be used to study circadian regulation of these different physiological processes and provide an understanding of the circadian system as a whole. This chapter outlines studies directed towards circadian control of metabolism in Drosophila.

\section{Use of Drosophila to Study Behavior and Metabolic Function}

As noted above, Drosophila has proved to be an outstanding system to dissect the molecular basis of the clock. Genes first found in Drosophila are now known to be mutated in some human circadian disorders. It is now also clear that Drosophila can be exploited to provide a complete understanding of the neural circuits that drive rhythms in behavior. The per and tim mutants were isolated through screens that used eclosion behavior as an assay for circadian function. Eclosion is "gated" by the circadian clock to occur around dawn, so while it only occurs once in the life of every fly, it can be monitored as a rhythm in a population. In addition to eclosion, the per and tim mutants were found to affect rhythms of rest:activity, and subsequently, in particular with the development of high throughput systems for monitoring locomotor activity, the field shifted to almost exclusively using rest:activity as a readout of internal clock function. Through work done in several laboratories, we now have a fairly good understanding of the clock neurons in the brain that drive rhythms of rest:activity (Nitabach and Taghert 2008). Interestingly, different subsets of neurons are required for different aspects of the overt rhythm, for instance. for the morning and evening peaks of locomotor activity. In addition, we recently identified a neural circuit that connects the clock neurons to other brain cells required for rhythmic rest:activity (Cavanaugh et al. 2014). It seems likely that, in the near future, we will be able to trace the passage of time-of-day signals all the way from the clock to the motor neurons that drive activity. 
Until 2008, little to no work had been done on circadian metabolism in Drosophila. However, flies have been used for general studies of metabolism, and are particularly useful as a model for aging, which is influenced strongly by metabolic parameters (Katewa and Kapahi 2010). As circadian regulation may be relevant for aging, we undertook to address links between metabolism and the circadian system.

\section{The Drosophila Fat Body Contains a Clock that Regulates a Rhythm of Feeding}

As we were accustomed to monitoring behavior in Drosophila, our studies of metabolic function also started with measurements of a metabolism-influenced behavior. We assayed food intake at different times of day and found that flies display a circadian rhythm of feeding such that food intake occurs maximally in the morning hours (Xu et al. 2008). A later study identified an additional peak of feeding that occurs later in the day and confirmed that nighttime hours of quiescence are associated with reduced food intake. As required of an endogenously driven rhythm, the rhythm of feeding persists in the dark, i.e., in the absence of environmental cycles. Also, it is eliminated in the dark in flies lacking the $C l k$ gene, demonstrating that it is under the control of the molecular clock mechanism described above (Xu et al. 2008).

To address the regulation of the feeding rhythm, we considered a role for the fat body, as this is a major metabolic tissue in Drosophila and is generally considered the functional equivalent of the liver. We found that clock genes, specifically tim, were expressed in the fat body and displayed a daily rhythm (Xu et al. 2008). To determine if this cycling was driven by a clock in the fat body, as opposed to signals from elsewhere, we disrupted the fat body clock by transgenically expressing a dominant negative version of the CLK protein. This manipulation abolished tim cycling, indicating that it depends upon a clock in the fat body. Interestingly, disruption of the fat body clock also affected the phase of the feeding rhythm, such that flies now showed maximal food consumption in the evening hours (Xu et al. 2008). The fact that the feeding rhythm was not abolished suggests that clocks in other tissues can also drive this rhythm.

\section{Fat Body and Neuronal Clocks Coordinately Regulate Metabolic Parameters}

We found that loss of the fat body clock did not just affect the feeding rhythm but also overall food intake (Xu et al. 2008). Food consumption was higher at all times of day relative to controls. Reasoning that increased food consumption increases sources of energy and therefore might be protective in adverse conditions of low 
nutrient availability, we tested flies lacking a fat body clock in starvation assays. To our surprise, we found that they were actually more sensitive to starvation and so died earlier than their wild type counterparts. This finding suggested that the increased food consumption was not increasing nutrient stores but was perhaps occurring in response to low endogenous levels of nutrients. Indeed, we found that glycogen and triglyceride levels were low in flies that lacked a clock in the fat body.

These results were unexpected because clock mutants, in other words flies lacking clocks in all tissues, do not show obvious metabolic phenotypes. The defects seen when only the fat body clock was ablated suggested that clocks in other tissues might have opposing effects on metabolic parameters. Neurons appeared to be good candidates for housing such clocks, as the brain is known to regulate metabolic activity, and so we disrupted clock function in neurons. We used the same tool as for the fat body clock (dominant negative clock proteins) and confirmed that neuronal clocks were disrupted by monitoring rest:activity behavior. As expected, rest:activity was arrhythmic. Measurement of metabolic parameters showed that nutrient stores, triglycerides and glycogen, were higher in flies with disrupted neuronal clocks than in wild type controls (Xu et al. 2008). As might be predicted, loss of neuronal clocks also increased resistance to starvation.

These data indicate that the fat body and the neuronal clock oppose each other in the control of metabolic function (Fig. 1). Typically, the fat body clock suppresses feeding, promotes storage of nutrients and increases resistance to starvation. Thus, loss of the fat body clock results in increased feeding, lower nutrient stores and sensitivity to starvation. Conversely, neurons are very metabolically active, and so clocks in these promote feeding, depletion of energy stores and sensitivity to starvation. All these functions are likely reversed when neuronal clocks are lost.

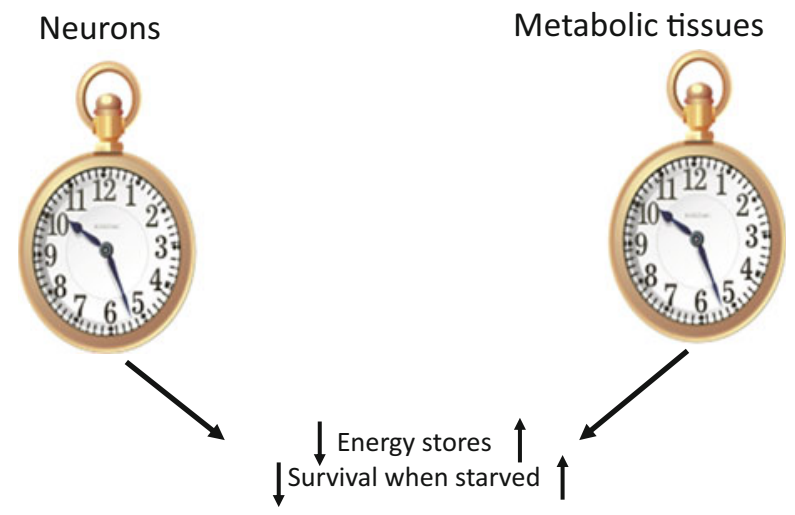

Fig. 1 Neuronal and metabolic clocks have opposing effects on metabolic parameters. These effects are predicted based upon phenotypes obtained by disrupting neuronal or fat body (metabolic) clocks. Disruption of neuronal clocks increases glycogen and trigyceride stores and promotes survival in response to starvation, whereas disruption of the fat body clock decreases glycogen and trigyceride stores, increases feeding and decreases survival upon starvation 
We documented increased nutrients and resistance to starvation in the absence of neuronal clocks but were unable to reliably quantify food intake, as this was so low.

In a subsequent study, we identified a specific group of neuron that regulate triglyceride levels (DiAngelo et al. 2011). These are the central clock neurons in the brain, which are critical for rest:activity rhythms. Interestingly, though, the effects of these neurons on triglyceride levels are separable from their effects on rest: activity.

\section{Rhythmic Gene Expression in the Fat Body Is Controlled Largely, but not Exclusively, by the Fat Body Clock}

To address the mechanisms by which the fat body clock regulates metabolic homeostasis, we sought to identify the genes expressed rhythmically in this tissue (Fig. 2). To this end, we collected tissue every $2 \mathrm{~h}$ around the clock over a 2-day period and profiled gene expression using microarrays (Xu et al. 2011). Simultaneously, we collected samples every $4 \mathrm{~h}$ from flies lacking a fat body clock due to expression of a dominant negative form of the CLK protein. We found that expression of many genes is cyclic in the fat body. Interestingly, several of these continue to cycle when the fat body clock is ablated, suggesting the influence of other factors, either the light:dark cycle or clocks elsewhere. In recent work, we have found that clocks in other tissues are required for at least some of the rhythmic cycling in the fat body.

Fig. 2 Circadian gene expression in the fat body: The protocol shown was followed to assay circadian gene expression in the fat body. Fat bodies were collected at 2 -h intervals over a 48 -h cycle in wild type flies and at 4-h intervals in flies lacking a fat body clock. Several classes of genes were found to cycle

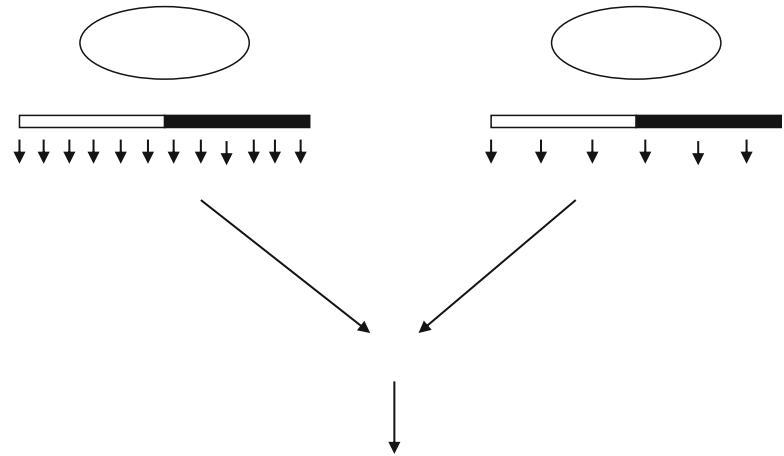

Microarrays 


\section{A Restricted Feeding Paradigm Resets the Phase of Cyclic Gene Expression in the Fat Body but not in the Brain}

The genes expressed cyclically in the fat body fall into many different functional categories, including lipid synthesis (in particular, fatty acid elongation), lipid breakdown, steroid hormone metabolism and immune function. The peak of gene expression for these different processes tended to occur at different times of day. To determine if temporal separation of gene expression by the clock was important for metabolic physiology, we sought to disrupt this temporal relationship. Reasoning that the time of feeding might be important for the peak in metabolic gene expression, but perhaps not for expression of immune genes, we restricted food to a time of day when feeding was typically less ( $6 \mathrm{~h}$ in the early evening) and we examined circadian gene expression ( $\mathrm{Xu}$ et al. 2011). We found that the time of feeding was indeed important, in fact even more than predicted. Thus, the clock in the fat body was reset by the time of feeding, which led to a reset of all downstream cycling genes.

Restricted feeding (RF) only changed the phase of gene expression if it occurred at the wrong time of day. If food was restricted to a time that corresponded to the normal daily peak of feeding, then the phase was maintained and the amplitude of the rhythm became stronger (note that normally the amplitude is low in constant darkness). On the other hand, RF had no effect on circadian gene expression in the brain (Xu et al. 2011).

\section{Decoupling Peripheral and Brain Tissues Decreases Reproductive Fitness}

As discussed above, a RF paradigm desynchronizes brain and fat body clocks as it resets the fat body, but not the brain clock. To determine if this process had physiological consequences, we monitored egg laying as a measure of reproductive fitness in animals maintained on RF. To exclude any influence of the duration of feeding, we compared egg production by flies fed for $6 \mathrm{~h}$ daily at the time they would normally eat with those fed for $6 \mathrm{~h}$ at the wrong time of day (Xu et al. 2011). Measurements of food intake showed equal food consumption in both groups, indicating that $18 \mathrm{~h}$ of starvation promoted equivalent feeding regardless of circadian time.

We found that flies fed at the wrong time laid fewer eggs than those fed at the correct time. However, these differences were not noted in a Clk mutant, indicating that they reflected an interaction of the time of feeding with endogenous clocks (Xu et al. 2011). We surmise that desynchrony of brain and peripheral clocks, achieved by an RF paradigm, reduced reproductive success. 


\section{Metabolic Signals Also Affect Clocks in the Brain}

While this chapter focuses on the circadian control of metabolism, we have also uncovered effects of metabolic signals of central clock function and rest:activity behavior. We found that the FOXO protein, a well-known component of metabolic pathways, is expressed in the fat body but can influence the brain clock's response to oxidative stress (Zheng et al. 2007). We also found that manipulations of the TOR-Akt pathway alter periodicity of rest:activity rhythms in parallel with effects on the molecular clock in brain neurons (Zheng and Sehgal 2010). Thus, metabolism and circadian clock interact on multiple levels, with consequences in both directions.

Open Access This chapter is distributed under the terms of the Creative Commons AttributionNoncommercial 2.5 License (http://creativecommons.org/licenses/by-nc/2.5/) which permits any noncommercial use, distribution, and reproduction in any medium, provided the original author(s) and source are credited.

The images or other third party material in this chapter are included in the work's Creative Commons license, unless indicated otherwise in the credit line; if such material is not included in the work's Creative Commons license and the respective action is not permitted by statutory regulation, users will need to obtain permission from the license holder to duplicate, adapt or reproduce the material.

\section{References}

Bargiello TA, Jackson FR, Young MW (1984) Restoration of circadian behavioural rhythms by gene transfer in Drosophila. Nature 312:752-754

Cavanaugh DJ, Geratowski JD, Wooltorton JR, Spaethling JM, Hector CE, Zheng X, Johnson EC, Eberwine JH, Sehgal A (2014) Identification of a circadian output circuit for rest:activity rhythms in Drosophila. Cell 157:689-701

DiAngelo JR, Erion R, Crocker A, Sehgal A (2011) The central clock neurons regulate lipid storage in Drosophila. PLoS One 6:e19921

Giebultowicz JM, Stanewsky R, Hall JC, Hege DM (2000) Transplanted Drosophila excretory tubules maintain circadian clock cycling out of phase with the host. Curr Biol 10:107-110

Jackson FR, Bargiello TA, Yun SH, Young MW (1986) Product of per locus of Drosophila shares homology with proteoglycans. Nature 320:185-188

Katewa SD, Kapahi P (2010) Dietary restriction and aging, 2009. Aging Cell 9:105-112

Konopka RJ, Benzer S (1971) Clock mutants of Drosophila melanogaster. Proc Natl Acad Sci USA 68:2112-2116

Krupp JJ, Billeter JC, Wong A, Choi C, Nitabach MN, Levine JD (2013) Pigment-dispersing factor modulates pheromone production in clock cells that influence mating in drosophila. Neuron 79:54-68

Liu X, Lorenz L, Yu QN, Hall JC, Rosbash M (1988) Spatial and temporal expression of the period gene in Drosophila melanogaster. Genes Dev 2:228-238

Myers EM, Yu J, Sehgal A (2003) Circadian control of eclosion. Interaction between a central and peripheral clock in Drosophila melanogaster. Curr Biol 13:526-533

Nitabach MN, Taghert PH (2008) Organization of the Drosophila circadian control circuit. Curr Biol 18:R84-R93 
Plautz JD, Kaneko M, Hall JC, Kay SA (1997) Independent photoreceptive circadian clocks throughout Drosophila. Science 278:1632-1635

Reddy P, Zehring WA, Wheeler DA, Pirrotta V, Hadfield C et al (1984) Molecular analysis of the period locus in Drosophila melanogaster and identification of a transcript involved in biological rhythms. Cell 38:701-710

Saez L, Young MW (1988) In situ localization of the per clock protein during development of Drosophila melanogaster. Mol Cell Biol 8:5378-5385

Sehgal A, Price JL, Man B, Young MW (1994) Loss of circadian behavioral rhythms and per RNA oscillations in the Drosophila mutant timeless. Science 263:1603-1606

Sehgal A, Rothenfluh-Hilfiker A, Hunter-Ensor M, Chen Y, Myers MP, Young MW (1995) Rhythmic expression of timeless: a basis for promoting circadian cycles in period gene autoregulation. Science 270:808-810

Xu K, Zheng X, Sehgal A (2008) Regulation of feeding and metabolism by neuronal and peripheral clocks in Drosophila. Cell Metab 8:289-300

Xu K, DiAngelo JR, Hughes ME, Hogenesch JB, Sehgal A (2011) The circadian clock interacts with metabolic physiology to influence reproductive fitness. Cell Metab 13:639-654

Zehring WA, Wheeler DA, Reddy P, Konopka RJ, Kyriacou CP et al (1984) P-element transformation with period locus DNA restores rhythmicity to mutant, arrhythmic Drosophila melanogaster. Cell 39:369-376

Zheng X, Sehgal A (2008) Probing the relative importance of molecular oscillations in the circadian clock. Genetics 178:1147-1155

Zheng X, Sehgal A (2010) AKT and TOR signaling set the pace of the circadian pacemaker. Curr Biol 20:1203-1208

Zheng X, Sehgal A (2012) Speed control: cogs and gears that drive the circadian clock. Trends Neurosci 35:574-585

Zheng X, Yang Z, Yue Z, Alvarez JD, Sehgal A (2007) FOXO and insulin signaling regulate sensitivity of the circadian clock to oxidative stress. Proc Natl Acad Sci USA 104: 15899-15904 